\title{
Immunogenicity Specimen Assessments Domain
}

National Cancer Institute

\section{Source}

National Cancer Institute. Immunogenicity Specimen Assessments Domain. NCI

Thesaurus. Code C112320.

A findings about domain for submitting dermal responses to antigens. 